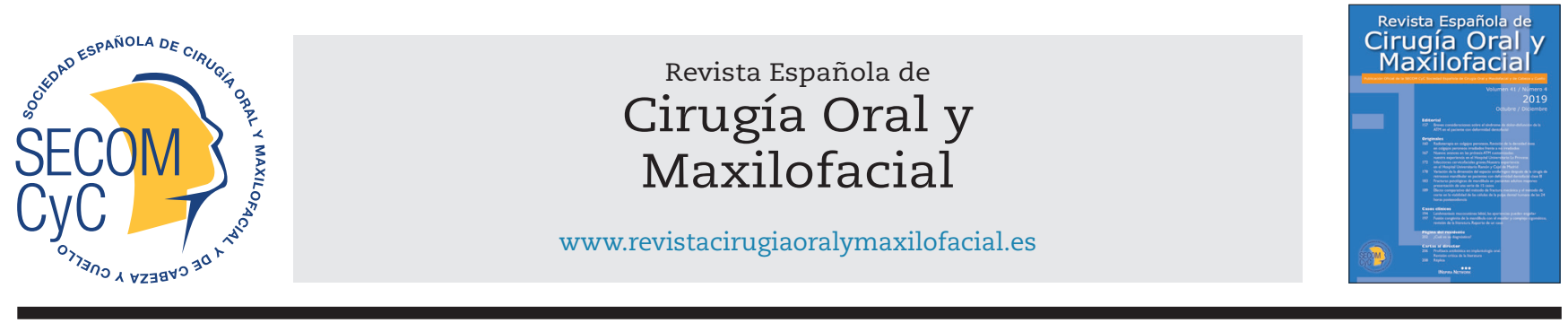

\title{
Editorial
}

\section{Breves consideraciones sobre el síndrome de dolor-disfunción de la ATM en el paciente con deformidad dentofacial}

En las pasadas IV Jornadas Nacionales de Articulación Temporomandibular (ATM), celebradas en Madrid los días 7 y 8 de noviembre de 2019, se abordó la patología de la ATM en los pacientes con deformidades dentofaciales. Los asistentes tuvieron ocasión de asistir a las ponencias de prestigiosos ponentes nacionales e internacionales, expertos en este campo, y pudieron profundizar en las diferentes opciones de tratamiento de aquel paciente que se somete a cirugía ortognática y padece, de forma concomitante, patología articular. Este editorial expone algunas breves consideraciones sobre este aspecto.

El tratamiento primario de la patología de la ATM tiene los siguientes objetivos: 1) aliviar los síntomas; 2) detener la progresión normal de la enfermedad, y 3) restaurar la normal función de la ATM; colocar el disco en su posición anatómica normal se considera controvertido al no existir aún una evidencia científica suficiente. Desde un punto de vista anatómico, el síndrome de dolor-disfunción de la ATM (SDDATM) (o trastorno interno de la ATM) se considera la relación anormal del disco articular con respecto al cóndilo mandibular, la eminencia articular y la fosa glenoidea del hueso temporal. Sin embargo, existen estudios que avalan la no completa correlación entre patología y posición discal: Kircos y cols. ${ }^{1}$ refirieron que un 34 \% de voluntarios asintomáticos presentaban desplazamiento discal, mientras que, de forma complementaria, Tallents y cols. ${ }^{2}$ observaron que el disco mantenía su posición normal en un 16-23 \% de los pacientes (sintomáticos).

La recidiva tras la osteotomía sagital de rama mandibular puede ocurrir por malposición condilar, pérdida de fijación y resorción condilar. La resorción condilar es un proceso lento que aparece tardíamente y que puede estar asociado con síntomas articulares; suele aparecer en pacientes con clase II dentoesquelética sometidos a avance mandibular con rotación antihoraria, habiéndose asociado igualmente a la existencia de mordida abierta anterior, mordida cruzada posterior y overjet maxilar extremo. Jung y cols., ${ }^{3}$ en un estudio retrospectivo sobre 460 pacientes tratados mediante cirugía ortognática, evaluaron la posición del disco articular pre y postoperatoriamente por medio de resonancia magnética nuclear (RMN); concluyeron que la severidad del desplazamiento discal se incrementaba progresivamente desde las deformidades de clase III a las de clase II en el plano sagital, y desde las deformidades hipodivergentes a las hiperdivergentes en el plano vertical, indicando una asociación entre clase II dentoesquelética y/o deformidad hiperdivergente y la presencia de desplazamiento discal, siendo difícil determinar si la primera es consecuencia de la segunda, o viceversa.

Existe cierta controversia en relación con la influencia del estado de la ATM en los pacientes que se someten a cirugía ortognática. Dujoncquoy y cols., ${ }^{4}$ en un estudio retrospectivo basado en encuestas a 57 pacientes, concluyen que los pacientes con disfunción de ATM que se someten a cirugía ortognática mejoran sus signos (74 \% para chasquidos, 27 \% para bloqueo articular) y síntomas (44 \% para dolor) de forma significativa; sin embargo, también observan que la cirugía ortognática puede originar dolor (24\%), chasquido articular (25\%) y bloqueo articular (17\%) en pacientes previamente asintomáticos. La revisión sistemática de Te Veldhuis y cols. ${ }^{5}$ sobre 76 artículos con 3399 pacientes y 380 controles, concluye que la gran variedad de técnicas quirúrgicas, criterios diagnósticos y técnicas de imagen, hace difícil comparar estudios para obtener conclusiones, teniendo todos ellos niveles de evidencia moderados o bajos (entre 2 y 4).

En relación con el manejo de estos pacientes, en 2007, Larry Wolford refirió el uso combinado de cirugía abierta de la ATM (por medio de meniscopexia con una miniancla de Mitek ${ }^{\mathrm{TM}}$ ) y cirugía ortognática simultáneamente, de forma segura y predecible, siendo necesarios un diagnóstico y tratamiento correctos y exigiendo al cirujano amplia experiencia en ambos tipos de cirugía ${ }^{6}$. Llamativos son los resultados de dos de sus estudios ${ }^{7,8}$, en los que obtiene resultados significativamente superiores en términos de dolor tras cirugía, dolor severo, resultado esquelético-oclusal y máxima apertura oral para el grupo de pacientes que, teniendo sintomatología articular prequirúrgica, fue intervenido mediante cirugía de la ATM de forma simultánea a la cirugía de la 
deformidad dentofacial, en comparación con los malos resultados del grupo al que solo se le practicó cirugía ortognática. En un reciente artículo de revisión, $\mathrm{Nale}^{9}$ sintetiza el estado del arte: 1) se aboga por que la enfermedad de la ATM y las deformidades dentofaciales sean tratadas de forma independiente una de la otra; 2) la mayoría de los autores proponen que primeramente se realice el tratamiento de la enfermedad articular, y 3) solo una vez que los síntomas articulares han mejorado debe realizarse el tratamiento quirúrgico de la maloclusión esquelética.

Nuestra filosofía en el manejo del paciente con sintomatología articular (dolor y/o dificultad para la apertura oral) es coincidente con la de Wolford en la necesidad imperativa del tratamiento quirúrgico de la patología articular (y por su alta prevalencia dentro de esta, de la del SDDATM) para obtener resultados predecibles en el paciente que se somete a cirugía ortognática, si bien consideramos que la cirugía mínimamente invasiva de la ATM por medio de artroscopia operativa (miotomía anterior del músculo pterigoideo lateral -para la liberación anterior del disco- y electrocoagulación del ligamento posterior por radiofrecuencia -para el tratamiento de la sinovitis y escarificación de la zona bilaminar-) es eficaz en el tratamiento del paciente sintomático (con dolor y/o limitación para la apertura oral), de forma previa a la cirugía de la deformidad dentofacial y como alternativa a la cirugía abierta de la ATM, cuyo concurso, eso sí, por medio de meniscopexia o meniscectomía, es incuestionable en casos refractarios al tratamiento artroscópico. La Figura 1 esquematiza nuestra propuesta de árbol de decisión.

A modo de sucinto resumen: 1) existe una disparidad manifiesta en lo publicado acerca del manejo del paciente con enfermedad de la ATM que va a ser sometido a cirugía ortognática para el tratamiento de la deformidad dentofacial; 2) creemos que el paciente con patología articular y deformidad dentofacial debe recibir tratamiento quirúrgico para ambas de forma independiente, realizando la cirugía articular de modo previo o, alternativamente, de forma simultánea a la cirugía ortognática; 3) en nuestra experiencia, la artroscopia operativa de la ATM es una técnica eficaz en el tratamiento de la mayoría de los pacientes con síndrome de dolor-disfunción articular previo a la cirugía ortognática.

Raúl González-García Servicio de Cirugia Oral y Maxilofacial. Hospital Universitario de Badajoz, España. Director de Revista Española de Cirugía Oral y Maxilofacial

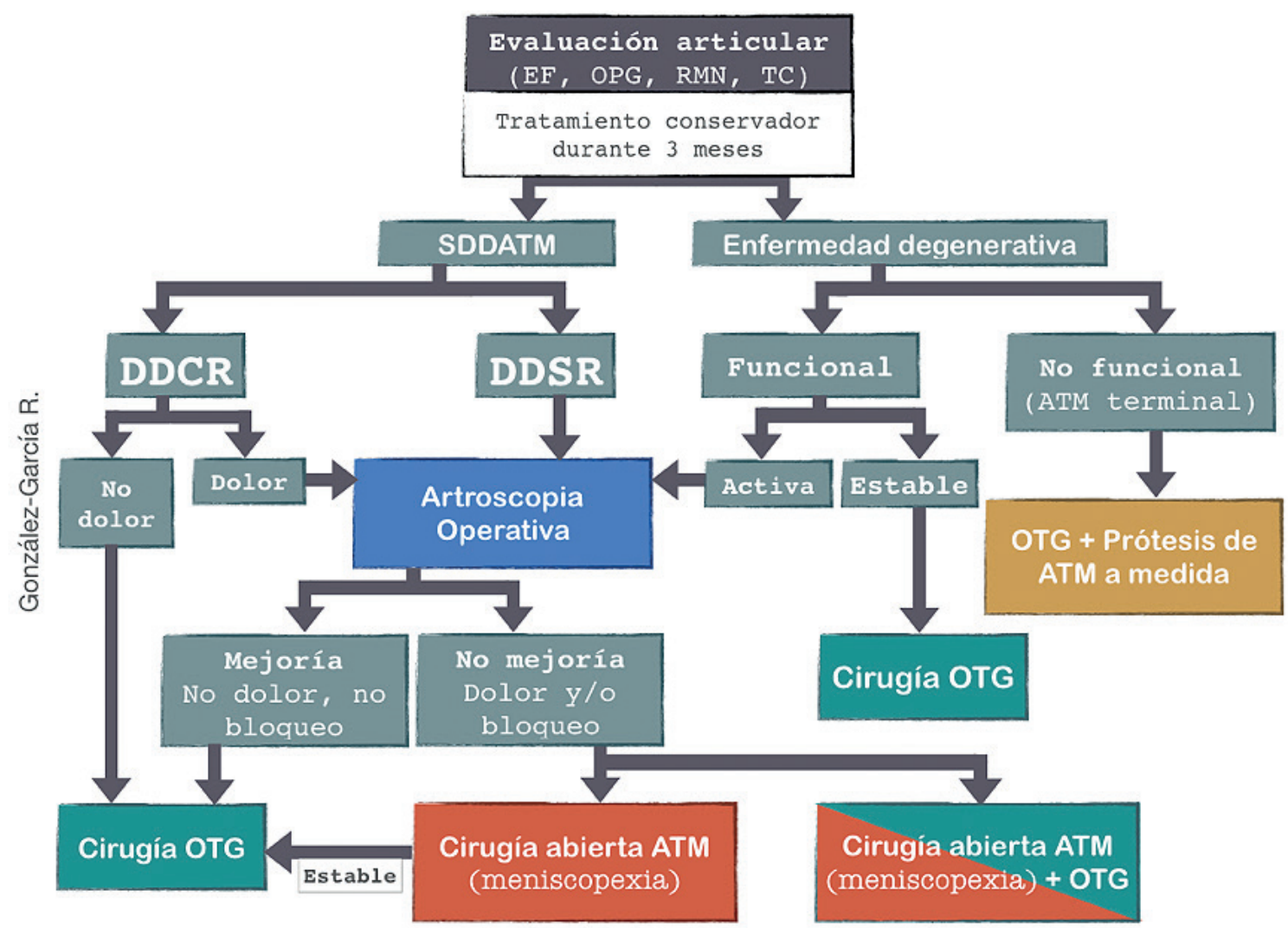

Figura 1. Propuesta de árbol de decisión terapéutica en el paciente con enfermedad articular y deformidad dentofacial concomitante. EF: exploración física. OPG: ortopantomografía. RMN: resonancia magnética nuclear. TC: tomografía computerizada. SDDATM: síndrome dolor-disfunción de ATM. DDCR: desplazamiento discal con reducción. DDSR: desplazamiento discal sin reducción. OTG: ortognática. 
B I B L I O G R A F Í A

1. Kircos LT, Ortendahl DA, Mark AS, Arakawa M. Magnetic resonance imaging of the TMJ disc in asymptomatic volunteers. J Oral Maxillofac Surg. 1987;45(10):852-4. DOI: 10.1016/0278-2391(87)90235-7.

2. Tallents RH, Katzberg RW, Murphy W, Proskin H. Magnetic resonance imaging findings in asymptomatic volunteers and symptomatic patients with temporomandibular disorders. J Prosthet Dent. 1996;75(5):529-33. DOI: 10.1016/s0022-3913(96)90458-8.

3. Jung WS, Kim H, Jeon DM, Mah SJ, Ahn SJ. Magnetic resonance imaging-verified temporomandibular joint disk displacement in relation to sagittal and vertical jaw deformities. Int J Oral Maxillofac Surg. 2013;42(9):1108-15. DOI: 10.1016/j.ijom.2013.03.012.

4. Dujoncquoy JP, Ferri J, Raoul G, Kleinheinz J. Temporomandibular joint dysfunction and orthognatic surgery: a retrospective study. Head Face Med. 2010;6:27. DOI: 10.1186/1746-160X-6-27.

5. Te Veldhuis EC, Te Veldhuis AH, Brammer WM, Wolvius EB, Koudstaal MJ. The effect of orthognatic surgery on the temporomandibular joint and oral function: a systematic review. Int J Oral Maxillofac Surg. 2017;46(5):554-63. DOI: 10.1016/j.ijom.2017.01.004.

6. Wolford LM. Clinical indications for simultaneous TMJ and orthognatic surgery. Cranio. 2007;25(4):273-82. DOI: 10.1179/crn.2007.041.

7. Wolford LM, Karras S, Mehra P. Concomitant temporomandibular joint and orthognatic surgery: a preliminary report. J Oral Maxillofac Surg. 2002;60(4):356-62. DOI: 10.1053/joms.2002.31220.

8. Wolford LM, Reiche-Fischel O, Mehra P. Changes in temporomandibular joint dysfunction after orthognatic surgery. J Oral Maxillofac Surg. 2003;61(6):655-60. DOI: 10.1053/joms.2003.50131.

9. Nale JC. Orthognatic surgery and the temporomandibular joint patient. Oral Maxillofac Surg Clin North Am. 2014;26(4):551-64. DOI: 10.1016/j.coms.2014.08.012. 